Article

\title{
An Expository Lecture of María Jesús Chasco on Some Applications of Fubini's Theorem
}

\author{
Alberto Castejón ${ }^{1, *}$, María Jesús Chasco ${ }^{2}$, Eusebio Corbacho ${ }^{1}$ and Virgilio Rodríguez de Miguel ${ }^{1}$ \\ 1 Departamento de Matemática Aplicada I, University of Vigo, 36310 Vigo, Spain; \\ eusebio.corbacho@gmail.com (E.C.); lvarela@uvigo.es (V.R.d.M.) \\ 2 Departamento de Física y Matemática Aplicada, University of Navarra, 31009 Pamplona, Spain; \\ mjchasco@unav.es \\ * Correspondence: acaste@uvigo.es
}

Citation: Castejón, A.; Chasco, M.J.; Corbacho, E.; de Miguel, V.R. An Expository Lecture of María Jesús Chasco on Some Applications of Fubini's Theorem. Axioms 2021, 10, 225. https://doi.org/10.3390/ axioms10030225

Academic Editors: Sidney A. Morris and Feliz Manuel Minhós

Received: 25 June 2021

Accepted: 30 August 2021

Published: 14 September 2021

Publisher's Note: MDPI stays neutral with regard to jurisdictional claims in published maps and institutional affiliations.

Copyright: (c) 2021 by the authors. Licensee MDPI, Basel, Switzerland. This article is an open access article distributed under the terms and conditions of the Creative Commons Attribution (CC BY) license (https:// creativecommons.org/licenses/by/ $4.0 /)$.

\begin{abstract}
The usefulness of Fubini's theorem as a measurement instrument is clearly understood from its multiple applications in Analysis, Convex Geometry, Statistics or Number Theory. This article is an expository paper based on a master class given by the second author at the University of Vigo and is devoted to presenting some Applications of Fubini's theorem. In the first part, we present Brunn-Minkowski's and Isoperimetric inequalities. The second part is devoted to the estimations of volumes of sections of balls in $\mathbb{R}^{n}$.
\end{abstract}

Keywords: Fubini's theorem; Brunn-Minkowski inequality; isoperimetric inequality; volumes of section of balls

MSC: 28A35; 52A40; 52A38

\section{Introduction}

Fubini's theorem and Brunn-Minkowski's inequality are two cornerstones of analytical methods in convex geometry with important applications to probability theory, partial differential equations and combinatorics. The present paper is an expository note on the subject based on a master class given by the second author at the University of Vigo some years ago. The aim of including it in this volume is to commemorate her teaching trajectory. We have tried to maintain the original exposition, other than removing some very easy facts from the original lecture. In this introduction, we intend to show that the subject is still interesting and to provide the reader with some useful references in order to explore the evolution of the subject until the present time.

The paper starts by recalling Fubini's theorem. After that, we give a detailed proof of Brunn-Minkowski's inequality and, as a corollary of it, the classical isoperimetric inequality which states that, among bodies of a given volume in $\mathbb{R}^{n}$, the Euclidean balls have the least surface area. This result appears to have been known in ancient times for two dimensions. By the end of the last century, there were a number of proofs which worked arbitrarily in many dimensions. It is interesting to remark that the formulation of the reverse isoperimetric problem needs some care because even convex bodies can have a large surface area and a small volume [1]. A big part of the classical BrunnMinkowski theory is concerned with establishing generalizations and analogues of the Brunn-Minkowski inequality for other geometric invariants. See the excellent survey article of Gardner [2] and the book of Schneider [3], which contains a comprehensive account of different aspects and consequences of Brunn-Minkowski inequality. More recent papers about Brunn-Minkowski-type inequalities include [4-7].

The second part of this paper is devoted to applying Fubini's theorem and BrunnMinkowski's inequality to obtain estimations of volumes of sections of balls in $\mathbb{R}^{n}$. The study of the geometry of convex bodies based on information about sections and projections 
of the bodies has important applications in many areas of science. The Fourier analytic approach to sections of convex bodies is based on certain formulas expressing the volume of sections in terms of the Fourier transform of powers of the Minkowski functional of a body. This approach was extended to obtain volumes of projections of convex bodies obtaining counterparts of the results of sections (see $[8,9])$.

In the study of convex bodies from a geometric and analytic point of view, some other basic questions appeared. One is about the distribution of the volume of high-dimensional convex bodies [10]. Moreover, in [11] the authors established the log-concavity of the volume of central sections of dilations of the cross-polytope $B_{1}^{n}$. Another remarkable paper on the subject is [12], where the maximal and minimal volume of non-central sections of the cross-polytope are obtained. There are also very recent, interesting results concerning sections of other convex bodies, such as the cube (see [13]).

\section{Preliminaries}

We recall in this section the concepts and notations used in the rest of the article. We will not go into great detail because they are elementary and can be found in any introductory book on Functional Analysis or Measure Theory (see for instance [14]).

If \|\| is a fixed norm in $\mathbb{R}^{n}$, the set $B=\left\{x \in \mathbb{R}^{n}:\|x\| \leq 1\right\}$ is called the unit ball. The dual space of $\mathbb{R}^{n}$ is the space of continuous linear forms endowed with the norm $\|f\|=\sup _{\|x\| \leq 1}|f(x)|$ and can be identified with $\mathbb{R}^{n}$. For a subset $B$ of $\mathbb{R}^{n}$, $\|x\|=\|x\|_{B}:=\inf \left\{\lambda>0: \lambda^{-1} x \in B\right\}$ denotes the Minkowski functional corresponding to the set $B$. Whenever you have a convex body $B$ in $\mathbb{R}^{n}$, that is, $B$ is a compact convex set with non-empty interior and symmetric, its Minkowski functional \|\|$_{B}$ defines a norm whose unit ball is $B$.

The unit ball for the normed spaces $\left(\mathbb{R}^{n},\|\|_{p}\right)$, where $1 \leq p<\infty$ and $\|x\|_{p}=$ $\left(\sum_{i=1}^{n}\left|x_{i}\right|^{p}\right)^{\frac{1}{p}}$ for all $x \in \mathbb{R}^{n}$, will be denoted by $\mathbb{B}_{p}^{n}=\left\{x \in \mathbb{R}^{n}\right.$ s.t $\left.\|x\|_{p} \leq 1\right\}$. In particular, when $p=2,\|\|_{2}$ is called the euclidean norm and it generates the euclidean topology in $\mathbb{R}^{n}$.

A measure space $(X, \mathcal{M}, \mu)$ is a triple formed by any set $X$, a $\sigma$-algebra $\mathcal{M}$ defined on its subsets and a measure $\mu$ defined on $\mathcal{M}$. Members of $\mathcal{M}$ are called measurable sets. A measure space is called sigma-finite if there exists a countable number $\left\{A_{n} n \in \mathbb{N}\right\}$ of measurable sets in $\mathcal{M}$ such that $X=\cup_{n \in \mathbb{N}} A_{n}$ and $\mu\left(A_{n}\right)<\infty$ for any $n \in \mathbb{N}$.

A map $f$ between two measure spaces $(X, \mathfrak{M}, \mu)$ and $(Y, \mathfrak{N}, v)$ is called measurable if $f^{-1}(B) \in \mathfrak{M} \forall B \in \mathfrak{N}$. Given two such measure spaces you can canonically construct the measure product space $(X \times Y, \mathfrak{M} \otimes \mathfrak{N}, \mu \times v) . \mathfrak{M} \otimes \mathfrak{N}$ is called the product $\sigma$-algebra of $\mathfrak{M}$ and $\mathfrak{N}$, and $\mu \times v$ the product measure of $\mu$ and $\nu$.

We are especially interested in the case where $X=\mathbb{R}^{n}, \mathcal{M}=\mathcal{M}_{n}$ is the Lebesgue $\sigma$-algebra in $\mathbb{R}^{n}$ and $\mu=m_{n}$ is the Lebesgue measure on $\mathcal{M}_{n}\left(m_{n}\right.$ is the completion of the product measure $m \times \ldots n$ times $\cdots \times m$, where $m$ is the Lebesgue measure on $\mathbb{R}$ ). This measure space is $\sigma$-finite. $\mathcal{M}_{n}$ properly contains the Borel $\sigma$-algebra $\mathcal{B}_{n}$ (generated by the open sets of the euclidean topology in $X=\mathbb{R}^{n}$ ). Moreover, the Lebesgue measure is a Radon measure: that is, all compact sets $K$ have finite measures, and it is outer and inner regular (for every Borel set, its measure is the infimum of the measures of the open sets containing it and for every open set its measure is the maximum of the measures of the compact sets contained in it, respectively). For a measurable set $A$, vol $(A)$, volume of $A$, will be just $m_{n}(A)$.

Our measurable functions will be defined on $\left(\mathbb{R}^{n}, \mathcal{M}_{n}, m_{n}\right)$ and will take real values in $(\mathbb{R}, \mathcal{M}, m)$. By $\int_{\mathbb{R}^{n}} f d m_{n}$, we denote the Lebesgue integral of a measurable function $f$. We say that $f$ is integrable if $\int_{\mathbb{R}^{n}}|f| d m_{n}<\infty$. The set of all integrable functions is a normed space denoted by $\mathbb{L}^{1}\left(\mathbb{R}^{n}\right)$ and $\|f\|_{1}=\int_{\mathbb{R}^{n}}|f| d m_{n}$. In the same way that $\mathbb{L}^{1}\left(\mathbb{R}^{n}\right)$, it can be defined as the normed space $\mathbb{L}^{p}\left(\mathbb{R}^{n}\right)$ for $1<p \in \mathbb{R}$ taking \|\|$_{p}$ as the norm defined by $\|f\|_{p}=\left(\int_{\mathbb{R}^{n}}|f|^{p} d m_{n}\right)^{\frac{1}{p}}$. We recall here the Dominated Convergence Theorem, which will be used later on: if $\left\{f_{n}\right\}$ is a sequence of measurable functions pointwisely convergent to a 
function $f$ and there exists an integrable $g$ such that $\left|f_{n}\right| \leq g \forall n \in \mathbb{N}$, then $f$ is integrable and the limit of the integrals of $f_{n}$ equals the integral of $f$.

In the computation of volumes it plays an important role in the Euler $\Gamma$-function, which is defined this way:

$$
\begin{array}{rlr}
\Gamma: \mathbb{R}_{+} & \rightarrow & \mathbb{R} \\
x & \mapsto \quad \int_{0}^{\infty} t^{x-1} e^{-t} d t,
\end{array}
$$

with the following property and values:

$$
\Gamma(x+1)=x \Gamma(x) \quad \forall x>0, \quad \Gamma(1)=1, \quad \Gamma\left(\frac{1}{2}\right)=\sqrt{\pi} .
$$

We finish this section with the statement of Fubini's theorem ([14], Theorem 8.8):

Theorem 1. Let $(X, \mathcal{M}, \mu),(Y, \mathcal{N}, v)$ be $\sigma$-finite measure spaces. Let $F: X \times Y \rightarrow \mathbb{R}$ be an $\mathcal{M} \times \mathcal{N}$-measurable function. Let us consider the functions:

$$
\begin{aligned}
\varphi^{*}: X & \longrightarrow[0, \infty) \\
x & \longrightarrow \int_{Y}|F(x, .)| d v
\end{aligned} \text { and } \begin{aligned}
\psi^{*}: Y & \longrightarrow[0, \infty) \\
y & \longrightarrow \int_{X}|F(., y)| d \mu \text {, then: }
\end{aligned}
$$

1. $\varphi^{*} \in \mathbb{L}^{1}(X, \mu) \Rightarrow F \in \mathbb{L}^{1}(X \times Y, \mu \times v)$.

2. $\psi^{*} \in \mathbb{L}^{1}(Y, v) \Rightarrow F \in \mathbb{L}^{1}(X \times Y, \mu \times v)$.

If $F \in \mathbb{L}^{1}(X \times Y, \mu \times v)$, then:

3. There is $E \subset X$ with $\mu(X \backslash E)=0$ such that $F(x,.) \in \mathbb{L}^{1}(Y, v) \forall x \in E$ and

$$
\begin{aligned}
\varphi: E & \longrightarrow \mathbb{R} \\
x & \longrightarrow \int_{Y} F(x, .) d v \text { is in } \mathbb{L}^{1}\left(E, \mu_{E}\right) .
\end{aligned}
$$

4. There is $G \subset Y$ with $v(Y \backslash G)=0$ such that $F(., y) \in \mathbb{L}^{1}(X, \mu) \forall y \in G$ and

$$
\begin{aligned}
\psi: G & \longrightarrow \mathbb{R} \\
y & \longrightarrow \int_{X} F(., y) d \mu \text { is in } \mathbb{L}^{1}\left(G, v_{G}\right) .
\end{aligned}
$$

Moreover,

$$
\int_{E} \varphi d \mu_{E}=\int_{X \times Y} F d(\mu \times v)=\int_{G} \psi d v_{G} .
$$

\section{Brunn-Minkowski's Inequality}

Next, we are going to use Fubini's theorem in the proof of Brunn-Minkowski inequality [15], which will be done by induction.

Theorem 2. If $A, B$ are compact sets in $\mathbb{R}^{n}$ with $n \geq 1$,

(1) $\forall \lambda \in[0,1], \quad \operatorname{vol}(\lambda A+(1-\lambda) B) \geq \operatorname{vol}(A)^{\lambda} \cdot \operatorname{vol}(B)^{1-\lambda}$

(2) $\operatorname{vol}(A+B) \geq\left(\operatorname{vol}(A)^{1 / n}+\operatorname{vol}(B)^{1 / n}\right)^{n} \quad($ Brunn -- Minkowski)

Proof. First step: (2) is consequence of (1).

In fact, taking

$$
\lambda=\frac{\operatorname{vol}(A)^{1 / n}}{\operatorname{vol}(A)^{1 / n}+\operatorname{vol}(B)^{1 / n}}
$$

and, considering the compact sets $A^{\prime}=\operatorname{vol}(A)^{-1 / n} \cdot A, B^{\prime}=\operatorname{vol}(B)^{-1 / n} \cdot B$, we have

$$
\begin{gathered}
\operatorname{vol}\left(\lambda A^{\prime}+(1-\lambda) B^{\prime}\right) \geq\left[\operatorname{vol}\left(\operatorname{vol}(A)^{-1 / n} A\right)\right]^{\lambda} \cdot\left[\operatorname{vol}\left(\operatorname{vol}(B)^{-1 / n} B\right)\right]^{1-\lambda} \\
=\left[\operatorname{vol}(A)^{-1} \cdot \operatorname{vol}(A)\right]^{\lambda}\left[\operatorname{vol}(B)^{-1} \cdot \operatorname{vol}(B)\right]^{1-\lambda}=1 .
\end{gathered}
$$

In other words:

$$
\operatorname{vol}\left(\frac{A+B}{\operatorname{vol}(A)^{1 / n}+\operatorname{vol}(B)^{1 / n}}\right) \geq 1
$$


and then

$$
\operatorname{vol}(A+B) \geq\left(\operatorname{vol}(A)^{1 / n}+\operatorname{vol}(B)^{1 / n}\right)^{n}
$$

Second step: (1) is a consequence of the following lemma

Lemma 1. Let $f, g, \varphi: \mathbb{R}^{n} \rightarrow[0, \infty]$ be measurable functions, such that for some $\lambda \in(0,1)$

$$
\varphi(\lambda r+(1-\lambda) s) \geq f(r)^{\lambda} \cdot g(s)^{1-\lambda}, \forall r, s \in \mathbb{R}^{n} .
$$

Then,

$$
\int_{\mathbb{R}^{n}} \varphi(x) d m_{n}(x) \geq\left(\int_{\mathbb{R}^{n}} f(x) d m_{n}(x)\right)^{\lambda}\left(\int_{\mathbb{R}^{n}} g(x) d m_{n}(x)\right)^{1-\lambda} .
$$

Indeed, taking

$$
\varphi=1_{\lambda A+(1-\lambda) B}, f=1_{A}, g=1_{B}
$$

(1) is obtained.

Third step: it is enough to prove the lemma for $\|f\|_{\infty}=\|g\|_{\infty}=1$.

In fact if the lemma holds for $\|f\|_{\infty}=\|g\|_{\infty}=1$, it will also be true (by linearity of the integral) for any pair of bounded functions $f, g$, just applying the lemma to

$$
\Phi=\frac{\varphi}{\|f\|_{\infty}^{\lambda}\|g\|_{\infty}^{1-\lambda}}, F=\frac{f}{\|f\|_{\infty}} \text { and } G=\frac{g}{\|g\|_{\infty}} .
$$

Fourth step: proof of the lemma for $\|f\|_{\infty}=\|g\|_{\infty}=1, n=1$.

For $0 \leq t<1$, whenever $f(x) \geq t, g(y) \geq t$, we will have

$$
\varphi(\lambda x+(1-\lambda) y) \geq f(x)^{\lambda} \cdot g(y)^{1-\lambda} \geq t .
$$

So,

$$
\{x \in \mathbb{R}, \varphi(x) \geq t\} \supset \lambda\{x \in \mathbb{R}, f(x) \geq t\}+(1-\lambda)\{x \in \mathbb{R}, g(x) \geq t\}
$$

Now, since for non-empty compact sets $A, B$ of $\mathbb{R}$, we have

$$
\begin{aligned}
& \{\min A\}+B \subset A+B \text { and } A+\{\max B\} \subset A+B \\
\Rightarrow & m(A+B) \geq m[(\{\min A\}+B) \cup(A+\{\max B\})] \\
= & m(\{\min A\}+B)+m(A+\{\max B\})=m(B)+m(A),
\end{aligned}
$$

by the regularity of Lebesgue's measure in $\mathbb{R}$, for the measurable sets $A=\lambda\{x \in \mathbb{R}, f(x) \geq t\}$ and $B=(1-\lambda)\{x \in \mathbb{R}, g(x) \geq t\}$ we have

$$
m\{x \in \mathbb{R}, \varphi(x) \geq t\} \geq \lambda m\{x \in \mathbb{R}, f(x) \geq t\}+(1-\lambda) m\{x \in \mathbb{R}, g(x) \geq t\} .
$$

Integrating with respect to $t$ in $\mathbb{R}^{+}$:

$$
\begin{aligned}
\int_{0}^{\infty} m\{x & \in \mathbb{R}, \varphi(x) \geq t\} d m(t) \\
& \geq \lambda \int_{0}^{\infty} m\{x \in \mathbb{R}, f(x) \geq t\} d m(t)+(1-\lambda) \int_{0}^{\infty} m\{x \in \mathbb{R}, g(x) \geq t\} d m(t) .
\end{aligned}
$$

The first integral is

$$
\int_{0}^{\infty}\left(\int_{\{x \in \mathbb{R}: \varphi(x) \geq t\}} d m(x)\right) d m(t)=\int_{\mathbb{R}}\left(\int_{0}^{\varphi(x)} d m(t)\right) d m(x)=\int_{\mathbb{R}} \varphi(x) d m(x) .
$$


In the same way, the second and third integrals are:

$$
\int_{\mathbb{R}} f(x) d m(x) \text { and } \int_{\mathbb{R}} g(x) d m(x) .
$$

So:

$$
\begin{aligned}
\int_{\mathbb{R}} \varphi(x) d m(x) \geq \lambda \int_{\mathbb{R}} f(x) d m(x)+(1-\lambda) & \int_{\mathbb{R}} g(x) d m(x) \\
& \left.\geq\left(\int_{\mathbb{R}} f(x) d m(x)\right)^{\lambda}\left(\int_{\mathbb{R}} g(x) d m(x)\right)^{1-\lambda}\right),
\end{aligned}
$$

where the last inequality comes from

$$
\lambda a+(1-\lambda) b \geq a^{\lambda} \cdot b^{1-\lambda}, \forall a, b>0,
$$

because $\ln (x)$ is concave.

Let $n>1$ and suppose the result is proved for $n-1$.

Take a fixed $y \in \mathbb{R}$ and define

$$
\begin{aligned}
\varphi_{y}: \mathbb{R}^{n-1} & \longrightarrow[0, \infty) \\
t & \longrightarrow \varphi(t, y) .
\end{aligned}
$$

Define $f_{y}, g_{y}$ analogously.

If $y_{0}, y_{1} \in \mathbb{R}$ are such that $y=\lambda y_{1}+(1-\lambda) y_{0}$, then $\forall r, s \in \mathbb{R}^{n-1}$ we have:

$$
\begin{aligned}
\varphi_{y}(\lambda r+(1-\lambda) s)=\varphi\left(\lambda\left(r, y_{1}\right)+\right. & \left.(1-\lambda)\left(s, y_{0}\right)\right) \\
& \geq\left(f\left(r, y_{1}\right)\right)^{\lambda} \cdot\left(g\left(s, y_{0}\right)^{1-\lambda}=\left(f_{y_{1}}(r)\right)^{\lambda} \cdot\left(g_{y_{0}}(s)\right)^{1-\lambda}\right.
\end{aligned}
$$

So, if we apply the induction hypothesis to $\varphi_{y}, f_{y_{1}}, g_{y_{0}}$, we get

$$
\int_{\mathbb{R}^{n-1}} \varphi_{y} d m_{n-1} \geq\left(\int_{\mathbb{R}^{n-1}} f_{y_{1}} d m_{n-1}\right)^{\lambda} \cdot\left(\int_{\mathbb{R}^{n-1}} g_{y_{0}} d m_{n-1}\right)^{1-\lambda}
$$

and applying again the result for $n=1$,

$$
\begin{aligned}
\int_{\mathbb{R}^{n}} \varphi d m_{n} & =\int_{\mathbb{R}}\left(\int_{\mathbb{R}^{n-1}} \varphi_{y} d m_{n-1}\right) d m(y) \\
& \geq\left[\int_{\mathbb{R}}\left(\int_{\mathbb{R}^{n-1}} f_{y_{1}} d m_{n-1}\right) d m(y)\right]^{\lambda} \cdot\left[\int_{\mathbb{R}}\left(\int_{\mathbb{R}^{n-1}} g_{y_{0}} d m_{n-1}\right) d m(y)\right]^{1-\lambda} \\
& =\left(\int_{\mathbb{R}^{n}} f d m_{n}\right)^{\lambda} \cdot\left(\int_{\mathbb{R}^{n}} g d m_{n}\right)^{1-\lambda} .
\end{aligned}
$$

\section{Isoperimetric Inequality}

Brunn-Minkowski's inequality allows us to easily obtain the isoperimetric inequality.

Theorem 3. Let $C$ be a convex body in $\mathbb{R}^{n}$ with $n \geq 2$, let $\partial(C)$ its border and $A(\partial(C))$ its surface area or perimeter,

$$
\begin{gathered}
\mathbb{B}_{2}^{n}=\left\{x \in \mathbb{R}^{n}:\|x\|_{2} \leq 1\right\} \text { and } \mathbb{S}^{n-1}=\left\{x \in \mathbb{R}^{n}:\|x\|_{2}=1\right\} \\
A(\partial(C)) \geq\left(\frac{\operatorname{vol}(C)}{\operatorname{vol}\left(\mathbb{B}_{2}^{n}\right)}\right)^{\frac{n-1}{n}} A\left(\mathbb{S}^{n-1}\right)
\end{gathered}
$$

(Among all convex bodies with fixed area, the maximum volume is attained by the spheres). 
Proof. Although it is difficult to give a notion of the perimeter or surface area (area for short) of a general compact, the convex ones are well approximated by polytopes and their area can be defined by continuity. Thus, we obtain a notion of area which coincides, for differentiable manifolds of class $C^{1}$, with that corresponding to the canonical measure.

If such definition is accepted, the area is obtained from the volume by the intuitive formula [16]:

$$
A(\partial(C))=\lim _{t \rightarrow 0} \frac{\operatorname{vol}\left(C+t \mathbb{B}_{2}^{n}\right)-\operatorname{vol}(C)}{t} .
$$

Using the Brunn-Minkowski's inequality,

$$
\operatorname{vol}\left(C+t \mathbb{B}_{2}^{n}\right) \geq\left(\operatorname{vol}(C)^{\frac{1}{n}}+\left(t \operatorname{vol}\left(\mathbb{B}_{2}^{n}\right)^{\frac{1}{n}}\right)^{n} \geq \operatorname{vol}(C)+n t \operatorname{vol}\left(\mathbb{B}_{2}^{n}\right)^{\frac{1}{n}} \operatorname{vol}(C)^{\frac{n-1}{n}}+o(t)\right.
$$

and so

$$
\begin{aligned}
A(\partial(C)) \geq \operatorname{nvol}\left(\mathbb{B}_{2}^{n}\right)^{\frac{1}{n}} \operatorname{vol}(C)^{\frac{n-1}{n}} & \\
& =\operatorname{nvol}\left(\mathbb{B}_{2}^{n}\right) \operatorname{vol}(C)^{\frac{n-1}{n}} \operatorname{vol}\left(\mathbb{B}_{2}^{n}\right)^{\frac{1}{n}-1}=A\left(\mathbb{S}^{n-1}\right)\left(\frac{\operatorname{vol}(C)}{\operatorname{vol}\left(\mathbb{B}_{2}^{n}\right)}\right)^{\frac{n-1}{n}} .
\end{aligned}
$$

The volume of convex bodies is related to the geometrical properties of the corresponding spaces. So its study is important in the local theory of Banach spaces [15]. Next, we will try to show how Fubini's theorem can be used in the estimation of volumes of sections of balls. We will see two illustrative theorems.

\section{Estimations of Volumes of Sections of Balls in $\mathbb{R}^{n}$}

In the sequel, a ball $B$ will be a symmetric convex body in $\mathbb{R}^{n}$.

If \|\|$_{B}$ is the Minkowski's functional associated with $B,\left(\mathbb{R}^{n},\|\|_{B}\right)$ is a Banach space whose unit ball is $B .\left(\mathbb{R}^{n},\|\|_{B}\right)$ is a Hilbert space if and only if $B$ is an ellipsoid.

If $E$ is a $k$-dimensional subspace of $\left(\mathbb{R}^{n},\|\|_{B}\right)$ and $E^{\perp}$ is the orthogonal complement of $E$, the section $E \cap B$ is the unit ball of the normed subspace $E$ and the projection $P_{E^{\perp}}(B)$ is the unit ball of the quotient normed space $\mathbb{R}^{n} / E$.

Theorem 4. [15]

$$
\left(\begin{array}{l}
n \\
k
\end{array}\right)^{-1} \operatorname{vol}(E \cap B) \operatorname{vol}\left(P_{E^{\perp}}(B)\right) \leq \operatorname{vol}(B) \leq \operatorname{vol}(E \cap B) \operatorname{vol}\left(P_{E^{\perp}}(B)\right)
$$

Proof. First step: $\operatorname{vol}(B)$ can be expressed as $\operatorname{vol}(B)=\int_{P_{E^{\perp}}(B)} \operatorname{vol}((x+E) \cap B) d m_{n-k}(x)$. By Fubini's theorem,

$$
\begin{aligned}
\operatorname{vol}(B)=m_{n}(B) & =\int_{E^{\perp}} m_{k}\{y \in E: x+y \in B\} d m_{n-k}(x) \\
= & \int_{E^{\perp}} \operatorname{vol}((x+E) \cap B) d m_{n-k}(x)=\int_{P_{E^{\perp}}(B)} \operatorname{vol}((x+E) \cap B) d m_{n-k}(x),
\end{aligned}
$$

because if $x \notin P_{E^{\perp}}(B),(x+E) \cap B=\varnothing$.

Second step: We obtain the inequality on the right $\operatorname{vol}(B) \leq \operatorname{vol}(E \cap B) \operatorname{vol}\left(P_{E^{\perp}}(B)\right)$

$$
E \cap B=\frac{1}{2}[((x+E) \cap B)+((-x+E) \cap B)]
$$

and

$$
\operatorname{vol}((x+E) \cap B)=\operatorname{vol}((-x+E) \cap B)) .
$$


Then applying Brunn-Minkowski's inequality, it yields

$$
\operatorname{vol}(E \cap B)^{\frac{1}{k}} \geq \frac{1}{2}\left[\operatorname{vol}((x+E) \cap B)^{\frac{1}{k}}+\operatorname{vol}((-x+E) \cap B)^{\frac{1}{k}}\right]=\operatorname{vol}((x+E) \cap B)^{\frac{1}{k}}
$$

and hence, using First Step, we obtain $\operatorname{vol}(B) \leq \operatorname{vol}(E \cap B) \operatorname{vol}\left(P_{E^{\perp}}(B)\right)$.

Third step: We obtain the inequality on the left.

If $x \in t P_{E^{\perp}}(B), 0 \leq t \leq 1$, then $x=t P_{E^{\perp}}(b)$ being $b \in B$ and $t b \in x+E$.

By convexity $t b+(1-t)(E \cap B) \subset B$, so $t b+(1-t)(E \cap B) \subset(x+E) \cap B$ and, being Lebesgue measure translation invariant

$$
\operatorname{vol}[(1-t)(E \cap B)] \leq \operatorname{vol}((x+E) \cap B)
$$

hence

$$
(1-t(x))^{k} \operatorname{vol}(E \cap B) \leq \operatorname{vol}((x+E) \cap B),
$$

where $t(x)$ represents the Minkowski functional of $P_{E^{\perp}}(B)$. Finally,

$$
\begin{aligned}
\operatorname{vol}(B) \geq \operatorname{vol}(E \cap B) & \int_{P_{E^{\perp}}(B)}(1-t(x))^{k} d m_{n-k}(x) \\
= & \operatorname{vol}(E \cap B) \int_{P_{E^{\perp}}(B)}\left(\int_{t(x)}^{1} k(1-t)^{k-1} d t\right) d m_{n-k}(x) \\
= & \operatorname{vol}(E \cap B) \int_{0}^{1} k(1-t)^{k-1}\left(\int_{t P_{E^{\perp}}(B)} d m_{n-k}\right)(x) d t \\
& =\operatorname{vol}(E \cap B) \operatorname{vol}\left(P_{E^{\perp}}(B)\right) \int_{0}^{1} k(1-t)^{k-1} t^{n-k} d t \\
& =\operatorname{vol}(E \cap B) \cdot \operatorname{vol}\left(P_{E^{\perp}}(B)\right) \cdot\left(\begin{array}{c}
n \\
k
\end{array}\right)^{-1} .
\end{aligned}
$$

The following lemma gives us an expression of the volumes of sections of balls in $\mathbb{R}^{n}$.

Lemma 2. Let $\left\{u^{1}, \ldots, u^{n-k}\right\}$ be an orthonormal basis of $E^{\perp},\|\cdot\|$ the norm associated with the ball $B$ and $E(\varepsilon)=\left\{x \in \mathbb{R}^{n}:\left|\left\langle x, u^{j}\right\rangle\right| \leq \varepsilon, 1 \leq j \leq n-k\right\}$.

Then,

$$
\Gamma\left(1+\frac{k}{p}\right) \operatorname{vol}(E \cap B)=\lim _{\varepsilon \rightarrow 0}(2 \varepsilon)^{k-n} \int_{E(\varepsilon)} e^{-\|x\|^{p}} d m_{n}(x), \quad p>0
$$

Proof. First step.

$$
\operatorname{vol}(E \cap B) \geq(2 \varepsilon)^{k-n} \operatorname{vol}(E(\varepsilon) \cap B), \forall \varepsilon>0
$$

and

$$
\operatorname{vol}(E \cap B)=\lim _{\varepsilon \rightarrow 0}(2 \varepsilon)^{k-n} \operatorname{vol}(E(\varepsilon) \cap B) .
$$

By Fubini's theorem,

$$
\begin{array}{r}
\operatorname{vol}(E(\varepsilon) \cap B)=\int_{E^{\perp}} m_{k}\{y \in E: x+y \in E(\varepsilon) \cap B\} d m_{n-k}(x) \\
=\int_{E^{\perp} \cap E(\varepsilon)} m_{k}\{y \in E: x+y \in B\} d m_{n-k}(x) \\
=\int_{P_{E^{\perp}}(E(\varepsilon))} \operatorname{vol}((x+E) \cap B) d m_{n-k}(x) .
\end{array}
$$


Then, doing the change of variable $x=\varepsilon z$,

$$
\begin{aligned}
&(2 \varepsilon)^{k-n} \operatorname{vol}(E(\varepsilon) \cap B)=2^{k-n} \int_{P_{E^{\perp}}(E(1))} \operatorname{vol}((\varepsilon z+E) \cap B) d m_{n-k}(z) \\
& \leq 2^{k-n} \operatorname{vol}(E \cap B) \int_{P_{E^{\perp}}(E(1))} d m_{n-k}(z)=\operatorname{vol}(E \cap B) .
\end{aligned}
$$

This last inequality allows us to apply the dominated convergence theorem and also obtain that

$$
\lim _{\varepsilon \rightarrow 0}(2 \varepsilon)^{n-k} \operatorname{vol}(E(\varepsilon) \cap B)=\operatorname{vol}(E \cap B)
$$

Second step: Obtaining the result.

$$
\begin{aligned}
& (2 \varepsilon)^{k-n} \int_{E(\varepsilon)} e^{-\|x\|^{p}} d m_{n}(x)=(2 \varepsilon)^{k-n} \int_{E(\varepsilon)}\left(\int_{\|x\|^{p}}^{+\infty} e^{-t} d t\right) d m_{n}(x) \\
= & (2 \varepsilon)^{k-n} \int_{0}^{\infty} e^{-t}\left(\int_{t^{\frac{1}{p}}} B \cap E(\varepsilon)\right. \\
= & (2 \varepsilon)^{k-n} \int_{0}^{\infty} e^{-t} \operatorname{vol}\left(t^{\frac{1}{p}} B \cap E(\varepsilon)\right) d t \\
= & \int_{0}^{\infty}\left(2 \varepsilon t^{\frac{-1}{p}}\right)^{k-n} e^{-t} t^{\frac{k}{p}} \operatorname{vol}\left(B \cap E\left(\varepsilon t^{\frac{-1}{p}}\right)\right) d t \\
\longrightarrow & \int_{0}^{\infty} \operatorname{vol}(B \cap E) t^{\frac{k}{p}} e^{-t} d t=\operatorname{vol}(B \cap E) \Gamma\left(1+\frac{k}{p}\right) .
\end{aligned}
$$

Remark 1. If $E=\mathbb{R}^{n}$, we have $\Gamma\left(1+\frac{n}{p}\right) \operatorname{vol}(B)=\int_{\mathbb{R}^{n}} e^{-\|x\|^{p}} d m_{n}(x)$, which for $B=\mathbb{B}_{p}^{n}$ allows us to easily compute $\operatorname{vol}\left(\mathbb{B}_{p}^{n}\right)$ because the integral $\int_{\mathbb{R}^{n}} e^{-\|x\|_{p}^{p}} d m_{n}(x)$ is transformed by Fubini's theorem into:

$$
\Pi_{i=1}^{n} \int_{\mathbb{R}} e^{-\left|x_{i}\right|^{p}} d x_{i}=\left(2 \int_{0}^{\infty} e^{-t^{p}} d t\right)^{n}=\left(2 \int_{0}^{\infty} e^{-s} s^{\frac{1}{p}} d s\right)^{n}=\left(2 \Gamma\left(1+\frac{1}{p}\right)\right)^{n}
$$

and so,

$$
m_{n}\left(\mathbb{B}_{p}^{n}\right)=\frac{\left(2 \Gamma\left(1+\frac{1}{p}\right)\right)^{n}}{\Gamma\left(1+\frac{n}{p}\right)}
$$

In particular,

$$
\begin{aligned}
m_{n}\left(\mathbb{B}_{1}^{n}\right) & =\frac{2^{n}}{n !} \\
m_{n}\left(\mathbb{B}_{2}^{2 k}\right)=\frac{\pi^{k}}{k !} \text { and } m_{n}\left(\mathbb{B}_{2}^{2 k+1}\right) & =\frac{\pi^{k}}{1 / 2(1+1 / 2) \ldots(k+1 / 2) .}
\end{aligned}
$$

From the above lemma, we will obtain the next Theorem. In order to do that we need two definitions:

Definition 1. Let

$$
\begin{aligned}
f: \mathbb{R}^{n} & \longrightarrow \mathbb{R} \\
x & \longrightarrow e^{-\left\|\alpha_{p} x\right\|_{p}^{p},}
\end{aligned}
$$

where $\alpha_{p}=2 \Gamma\left(1+\frac{1}{p}\right)$. We define the measure $\mu_{p}^{n}$ as $\mu_{p}^{n}(A)=\int_{A} f(x) d m_{n}(x)$.

So defined, $\mu_{p}^{n}$ turns out to be a probability measure with density $f(x)$ with respect to $m_{n}$, because precisely

$$
\int_{\mathbb{R}^{n}} e^{-\|x\|_{p}^{p}} d m_{n}(x)=\alpha_{p}^{n} .
$$


Definition 2. Let $\mu, v$ be Radon positive measures on $\mathbb{R}^{n}$. The measure $\mu$ is said to be finer than the measure $v(\mu \succ v)$, if for any ball $B \subset \mathbb{R}^{n}, \mu(B) \geq v(B)$.

Theorem 5. [17] If $1 \leq q \leq p<\infty$, $\frac{\operatorname{vol}\left(\mathbb{B}_{p}^{n} \cap E\right)}{\operatorname{vol}\left(\mathbb{B}_{p}^{k}\right)} \geq \frac{\operatorname{vol}\left(\mathbb{B}_{q}^{n} \cap E\right)}{\operatorname{vol}\left(\mathbb{B}_{q}^{k}\right) .}$

Proof. Applying the former lemma to $\mathbb{B}_{p}^{n}$, we have

$$
\operatorname{vol}\left(E \cap \mathbb{B}_{p}^{n}\right)=\frac{1}{\Gamma(1+k / p)} \lim _{\varepsilon \rightarrow 0}(2 \varepsilon)^{k-n} \int_{E(\varepsilon)} e^{-\|x\|_{p}^{p}} d m_{n}(x) .
$$

Changing the variables $x=\alpha_{p} z$

$$
\operatorname{vol}\left(E \cap \mathbb{B}_{p}^{n}\right)=\frac{\alpha_{p}^{k}}{\Gamma(1+k / p)} \lim _{\varepsilon \rightarrow 0}\left(\frac{2 \varepsilon}{\alpha_{p}}\right)^{k-n} \int_{E\left(\frac{\varepsilon}{\alpha_{p}}\right)} e^{-\left\|\alpha_{p} z\right\|_{p}^{p}} d m_{n}(z)
$$

and calling $\eta$ to $\frac{\varepsilon}{\alpha_{p}}$

$$
\operatorname{vol}\left(E \cap \mathbb{B}_{p}^{n}\right)=\operatorname{vol}\left(\mathbb{B}_{p}^{k}\right) \lim _{\eta \rightarrow 0}(2 \eta)^{k-n} \mu_{p}^{n}(E(\eta))
$$

or equivalently

$$
\frac{\operatorname{vol}\left(E \cap \mathbb{B}_{p}^{n}\right)}{\operatorname{vol}\left(\mathbb{B}_{p}^{k}\right)}=\lim _{\eta \rightarrow 0}(2 \eta)^{k-n} \mu_{p}^{n}(E(\eta))
$$

and analogously

$$
\frac{\operatorname{vol}\left(E \cap \mathbb{B}_{q}^{n}\right)}{\operatorname{vol}\left(\mathbb{B}_{q}^{k}\right)}=\lim _{\eta \rightarrow 0}(2 \eta)^{k-n} \mu_{q}^{n}(E(\eta)) .
$$

Let us see now that for $p \geq q, \mu_{p}^{1} \succ \mu_{q}^{1}$.

In fact, it is enough to see that $g(x)=\int_{0}^{x}\left(e^{-\left|\alpha_{p} t\right|^{p}}-e^{-\left|\alpha_{q} t\right|^{q}}\right) d t \geq 0, \forall x>0$ and this is so because $g(0)=0, g(\infty)=1 / 2-1 / 2=0, g^{\prime}(x)$ vanishes in one single point and moreover it is positive on a neighbourhood of 0 .

Moreover, if $\mu_{1} \succ v_{1}$ and $\mu_{2} \succ v_{2}$ being $\mu_{i}, v_{i}, i=1,2$ Radon positive measures with concave logarithm density with respect to $m_{s_{i}}$ in $\mathbb{R}^{s_{i}}$, for $i=1,2$, then $\mu_{1} \times \mu_{2} \succ v_{1} \times v_{2}$ in $\mathbb{R}^{s_{1}+s_{2}}[13]$.

Hence, if $p \geq q, \mu_{p}^{n} \succ \mu_{q}^{n}$.

Now, being $E(\eta)$ symmetric convex with non-empty interior and the measures $\mu_{p}^{n}, \mu_{q}^{n}$ regular and satisfying $\mu_{p}^{n} \succ \mu_{q}^{n}$, we have that $\mu_{p}^{n}(E(\eta)) \geq \mu_{q}^{n}(E(\eta))$ and so

$$
\frac{\operatorname{vol}\left(E \cap \mathbb{B}_{p}^{n}\right)}{\operatorname{vol}\left(\mathbb{B}_{p}^{k}\right)} \geq \frac{\operatorname{vol}\left(E \cap \mathbb{B}_{q}^{n}\right)}{\operatorname{vol}\left(\mathbb{B}_{q}^{k}\right)}
$$

We finish this note with some consequences:

Remark 2. Taking into account that $E \cap \mathbb{B}_{2}^{n}=\mathbb{B}_{2}^{k}$, we obtain from Theorem 5 :

$$
\begin{aligned}
& \text { For } 2 \leq p<\infty, \operatorname{vol}\left(E \cap \mathbb{B}_{p}^{n}\right) \geq \operatorname{vol}\left(\mathbb{B}_{p}^{k}\right) \\
& \text { For } 1 \leq p \leq 2, \operatorname{vol}\left(E \cap \mathbb{B}_{p}^{n}\right) \leq \operatorname{vol}\left(\mathbb{B}_{p}^{k}\right) .
\end{aligned}
$$

On the other hand, if $B, B^{\prime}$ are balls in $\mathbb{R}^{n}$ such that $B \subset B^{\prime}$, we obtain from Theorem 5 that:

$$
\frac{\operatorname{vol}\left(B^{\prime} \cap E\right)}{\operatorname{vol}\left(B^{\prime}\right)} \leq\left(\begin{array}{l}
n \\
k
\end{array}\right) \frac{1}{\operatorname{vol}\left(P_{E^{\perp}}\left(B^{\prime}\right)\right)} \leq\left(\begin{array}{l}
n \\
k
\end{array}\right) \frac{1}{\operatorname{vol}\left(P_{E^{\perp}}(B)\right)} \leq\left(\begin{array}{l}
n \\
k
\end{array}\right) \frac{\operatorname{vol}(B \cap E)}{\operatorname{vol}(B) .}
$$


In particular:

$$
\begin{aligned}
& \text { For } 2 \leq p \leq \infty, \operatorname{vol}\left(E \cap \mathbb{B}_{p}^{n}\right) \leq\left(\begin{array}{l}
n \\
k
\end{array}\right) \frac{\operatorname{vol}\left(\mathbb{B}_{2}^{k}\right)}{\operatorname{vol}\left(\mathbb{B}_{2}^{n}\right)} \operatorname{vol}\left(\mathbb{B}_{p}^{n}\right) \\
& \text { For } 1 \leq p \leq 2, \operatorname{vol}\left(E \cap \mathbb{B}_{p}^{n}\right) \geq\left(\begin{array}{l}
n \\
k
\end{array}\right)^{-1} \frac{\operatorname{vol}\left(\mathbb{B}_{2}^{k}\right)}{\operatorname{vol}\left(\mathbb{B}_{2}^{n}\right)} \operatorname{vol}\left(\mathbb{B}_{p}^{n}\right) .
\end{aligned}
$$

Author Contributions: Investigation, A.C., M.J.C., E.C. and V.R.d.M.; writing-original draft preparation, A.C., M.J.C., E.C. and V.R.d.M.; writing—review and editing, A.C., M.J.C., E.C. and V.R.d.M. All authors have read and agreed to the published version of the manuscript.

Funding: The second author was supported by Spanish Agencia Estatal de Investigación (AEI) grant MTM2016-79422-P cofinantiated by Fondo Europeo de Desarrollo Regional (FEDER, UE).

Data Availability Statement: No new data were created or analyzed in this study. Data sharing is not applicable to this article.

Conflicts of Interest: The authors declare no conflict of interest. The funders had no role in the design of the study; in the collection, analyses, or interpretation of data; in the writing of the manuscript; or in the decision to publish the results.

\section{References}

1. Ball, K. Convex geometry and functional analysis. Handb. Geom. Banach Spaces 2001, 1, 161-194.

2. Gardner, R.J. The Brunn-Minkowski inequality. Bull. Am. Math. Soc. 2002, 39, 355-405. [CrossRef]

3. Schneider, R. Convex Bodies: The Brunn-Minkowski Theory; Cambridge University Press: Cambridge, UK, 2014.

4. Böröczky, K.J.; Lutwak, E.; Yang, D.; Zhang, G. The log-Brunn-Minkowski inequality. Adv. Math. 2012, 231, 1974-1997. [CrossRef]

5. Böröczky, K.J.; Lutwak, E.; Yang, D.; Zhang, G. The logarithmic Minkowski problem. J. Am. Math. Soc. 2013, 26, 831-852. [CrossRef]

6. Ma, L. A new proof of the log-Brunn-Minkowski inequality. Geom. Dedicata 2015, 177, 75-82. [CrossRef]

7. Yang, Y.; Zhang, D. The log-Brunn-Minkowski inequality in $\mathbb{R}^{3}$. Proc. Am. Math. Soc. 2019, 147, 4465-4475. [CrossRef]

8. Barthe, F.; Naor, A. Hyperplane projections of the unit ball of $l_{p}$. Discret. Comput. Geom. 2002, 27, 215-226. [CrossRef]

9. Koldobsky, A. Fourier Analysis in Convex Geometry. Mathematical Surveys and Monogrhs (No. 116); American Mathematical Society: Providence, RI, USA, 2005.

10. Brazitikos, S.; Giannopoulos, A.; Valettas, P.; Vritsiou, B.H. Geometry of Isotropic Convex Bodies. Mathematical Surveys and Monograhs; American Mathematical Society: Providence, RI, USA, 2014; Volume 196.

11. Nayar, P.; Tkocz, T. On a convexity property of sections of the cross-polytope. Proc. Am. Math. Soc. 2020, 148, 1271-1278. [CrossRef]

12. Liu, R.; Tkocz, T. A note on the extremal non-central sections of the cross-polytope. Adv. Appl. Math. 2020, 118, 102031. [CrossRef]

13. König, H.; Rudelson, M. On the volume of non-central sections of a cube. Adv. Math. 2020, 360, 106929. [CrossRef]

14. Rudin, W. Real and Complex Analysis; McGraw-Hill Series in Higher Mathematics; McGraw-Hill: New York, NY, USA, 1987.

15. Pisier, G. The Volume of Convex Bodies and Banach Space Geometry; Cambridge Tracts in Mathematics; Cambridge University Press: Cambridge, UK, 1989; Volume 94.

16. Berger, M. Geometry I, II; Universitext; Springer: Berlin/Heidelberg, Germany, 1987.

17. Meyer, M.; Pajor, A. Sections of the unit ball of $\ell_{p}^{n}$. J. Funct. Anal. 1988, 80, 109-123. [CrossRef] 